TUMOUR SUPPRESSORS

\title{
Floxed foxes
}

DOI:

10.1038/nrc2094

\section{URLs}

Foxo1

http://www.ncbi.nlm.nih.gov/ entrez/query.fcgi?db=gene\&c $\mathrm{md}=$ Retrieve\&dopt=full_ report\&list_uids $=56458$

Foxo3

http://www.ncbi.nlm.nih.gov/ entrez/query.fcgi?db=gene\&c $\mathrm{md}=$ Retrievegdopt=full report\&list_uids $=56484$

Foxo4

http://www.ncbi.nlm.nih.gov/ entrez/query.fcgi?db=gene\&c $\mathrm{md}=$ RetrieveEdopt=full_ report\&list_uids $=54601$

\section{Spry2}

http://www.ncbi.nlm.nih.gov/ entrez/query.fcgi?db=gene\&c $\mathrm{md}=$ Retrievegdopt=full_ report\&list_uids $=24064$
Although a role for FOXO (forkhead box $\mathrm{O}$ ) family transcription factors as tumour suppressors has been proposed, direct evidence has been lacking. Ji-Hye Paik, Ron DePinho, Diego Castrillon and colleagues have shown that the deletion of all three FOXOs is necessary to induce a cancer-prone condition in mice.

To circumvent embryonic lethality, Paik et al. used an inducible Cre-lox system to knock out Foxo1, Foxo3 and Foxo4 in various combinations. The widespread somatic deletion of all three genes (Foxo1;3;4-null mice) caused thymic lymphomas and haemangiomas, which were associated with increased cell proliferation and survival in these lineages.

To investigate the mechanisms responsible for haemangioma development, the authors took advantage of the observation that despite the broad deletion of the FOXOs across the lineage, many, but not all, organs showed an increase in endothelial cell density. Transcriptional profiling identified genes that were differentially expressed in liver endothelial cells (a tissue in which haemangiomas developed) and lung endothelial cells (which did not develop haemangiomas) after FOXO deletion. They pared down the resulting list of 138 genes by examining the promoter regions for FOXO-binding elements, paying particular attention to those that contained binding elements that were conserved among three species (mouse, human and at least one other). They found 21 genes that contained at least one conserved FOXO binding element. The authors chose to focus on the gene with the most conserved FOXO binding elements, sprouty2 (Spry2), which is a negative regulator of receptor tyrosine kinases. Spry2 was validated as a direct FOXO target, and short hairpin RNA knockdown of sprouty2 protein in primary liver endothelial cell cultures increased cell-cycle progression and decreased apoptosis, similar to the phenotype of Foxo1;3;4-null endothelial cells. Also, further reduction of sprouty2 in Foxo1;3;4-null endothelial cells had no additional effect.

Although the tumour spectrum of Foxo 1;3;4-null mice is narrower than might have been expected, this genetic study indicates that these genes are tumour suppressors, and that FOXO functions in vivo are context-specific, depending on the cell type and tissue.

Sarah Seton-Rogers

ORIGINAL RESEARCH PAPER Paik, J. H. et al. FoxOs are lineage-restricted redundant tumour suppressors and regulate endothelial cell homeostasis. Cell 128, 309-323 (2007) FURTHER READING Tothova, Z. et al. FoxOs are critical mediators of hematopoietic stem cell resistance to physiologic oxidative stress. Cell 128, 325-339 (2007)

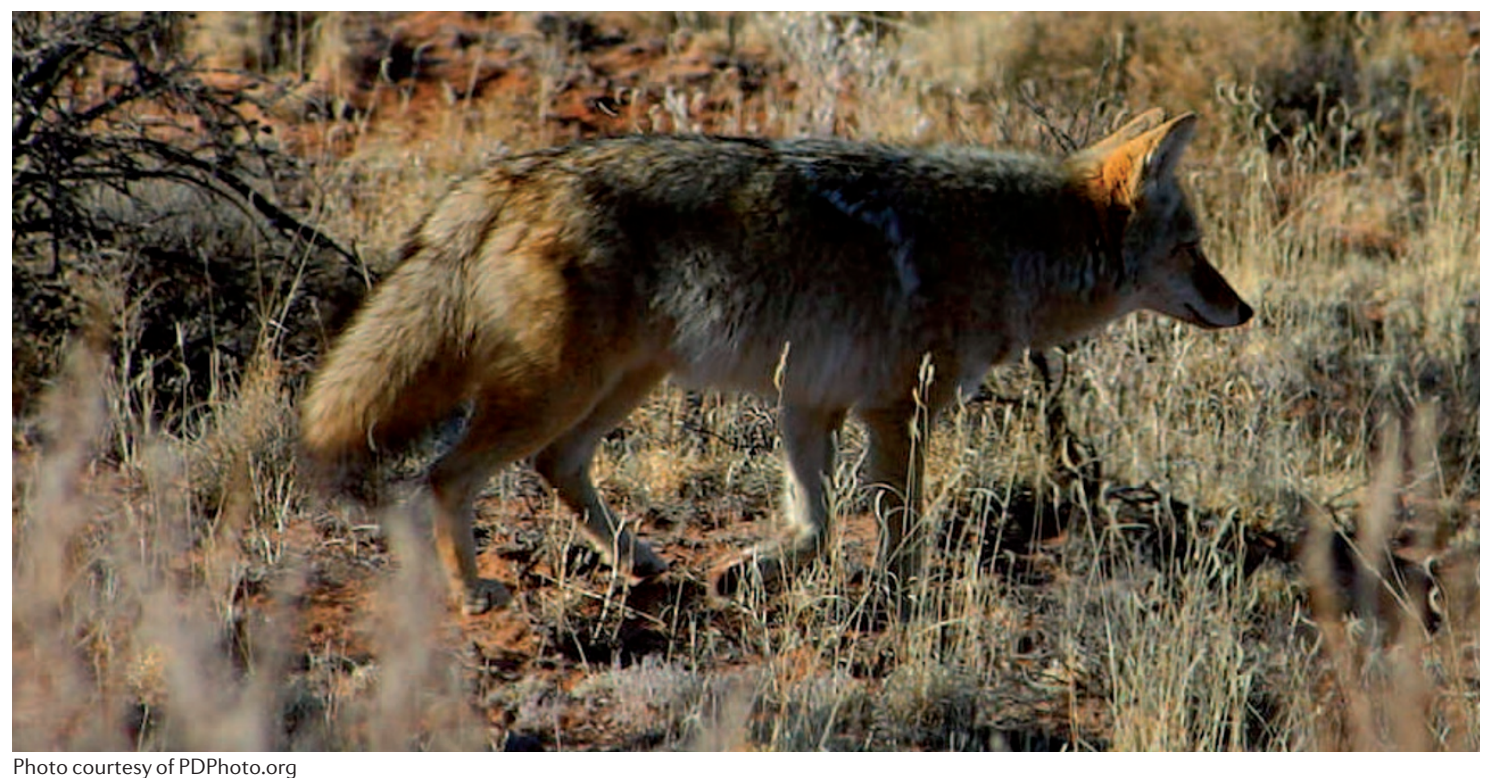

